\title{
Internal Medicine Residents' Perceptions of Pharmacist Involvement in Medical Rounds
}

Stephanie M. Tubb, PharmD ${ }^{1}$; Erin B. Loesch, PharmD, BCPS, DPLA ${ }^{2}$

${ }^{1}$ Dayton VA Medical Center, Dayton OH; Pharmacy Practice, Cedarville University School of Pharmacy

2 Dayton VA Medical Center, Dayton, $\mathrm{OH}$

\begin{abstract}
Background: Current physicians note the positive effects of clinical pharmacists on rounds, yet minimal evidence exists regarding medical residents' view of pharmacists in this setting. Knowing their perceptions of clinical pharmacists on acute care rounds will allow pharmacists to optimize their roles and improve their interprofessional interactions.

Objective: To assess internal medicine residents' perceptions of pharmacists on rounds, evaluate which recommendations they prefer to receive, and examine their past experiences with pharmacists on rounds.

Methods: Internal medicine residents were invited to complete an online survey containing 7 items regarding past experiences with pharmacists on rounds (5-point Likert-type scale; 1=Strongly Disagree, 5=Strongly Agree), 3 items about preferred recommendations (ranking questions), and 6 items regarding perceptions of pharmacy practice (5-point Likert-type scale; 1=Strongly Disagree, 5=Strongly Agree). Data were analyzed using frequencies.

Results: 27 residents participated (33.75\% response rate). A majority strongly agreed that they always want a pharmacist to be a part of their rounding team (Mean $\pm S D=4.93 \pm 0.26$ ). They prefer receiving recommendations from the pharmacist in-person before, during, or after rounds and appreciate recommendations on topics such as anticoagulants, antimicrobial stewardship, and renal dose adjustments. Residents did not express a strong knowledge of pharmacists' education and training processes $(M e a n \pm S D=3.77 \pm 1.05)$, which may have led to their lack of agreement that pharmacists are equipped to be mid-level practitioners (Mean $\pm S D=3.00 \pm 1.30)$. Conclusions: Internal medicine residents had positive experiences with rounding pharmacists and desire their involvement on rounds. Pharmacists should make recommendations to residents in-person and educate them on their education and training to allow for further advocacy for pharmacist services.
\end{abstract}

Keywords: medical residents, rounds, clinical pharmacist, perceptions

\section{Background}

Clinical pharmacists are pharmacists who are educated and trained in many direct patient care environments and who work closely with other healthcare professionals and patients to ensure that the medications prescribed will contribute to optimal health outcomes. ${ }^{1}$ There are multiple benefits to incorporating a clinical pharmacist into a medical rounding team, such as improved patient outcomes, shorter lengths of stay, and cost savings. By involving clinical pharmacists in medical ICU rounds, preventable adverse drug events can be decreased by up to $66 \% .^{2}$ Additionally, involving a clinical pharmacist in medication reconciliation, drug therapy plans, and discharge counseling on medical floors results in fewer patients transferred for more intensive care and shorter lengths of stay. ${ }^{3}$ One study found that incorporating a clinical pharmacist into a burn center's rounds resulted in a savings of $\$ 11,081.14$ over a 6 -month period from drug changes alone. ${ }^{4}$

Corresponding author: Stephanie M. Tubb, PharmD Clinical Pharmacy Specialist, Dayton VA Medical Center, Dayton OH; 4100 W Third Street (119), Dayton, OH 45428 Assistant Professor of Pharmacy Practice, Cedarville University School of Pharmacy; 251 North Main Street Cedarville, OH 45314; Email: scailor@cedarville.edu
Physicians have noticed the positive effects of having clinical pharmacists on rounds. A study by Abdel-Latif found that $74 \%$ of hospital physicians were willing to collaborate with a clinical pharmacist despite existing barriers such as physicians' poor knowledge of the clinical role of pharmacists and the lack of communication between their professions that hinder interprofessional collaboration. These hospital physicians expected clinical pharmacists to advise them on rational use of drugs, to resolve drug-related problems, and to counsel patients. ${ }^{5}$ Other studies have shown that physicians' perceptions of pharmacists' knowledge and competency in a rounding role factors into successful collaboration on rounds and that $31.7 \%$ of physicians did not expect pharmacists to be available for consultation during rounds. ${ }^{6,7}$

As future physicians, medical students' perceptions of pharmacy provide an inside look as to how rounding clinical pharmacists will be viewed in the future. Research has shown that medical students who have frequent (weekly or more) interaction with pharmacists have a significantly more positive perception of the clinical roles of pharmacists than those with infrequent interactions, although student involvement in interprofessional activities during medical school did not significantly impact their opinions on the role or qualification of pharmacists. ${ }^{8}$ While medical students may not interact with clinical pharmacists much during their years in school, these students will likely encounter a clinical pharmacist on the rounding team during their residency years. 
A study conducted in 2002 showed that physicians who had graduated from medical school less than 10 years prior had higher expectations of clinical pharmacists than those who had graduated over 10 years ago. ${ }^{9}$ The results of this study may be outdated, but there is no other evidence in the literature to infer how medical residents view clinical pharmacists on rounds. Knowing the medical residents' perceptions of clinical pharmacists on acute care rounds will allow these pharmacists to optimize their roles and improve their interprofessional interactions. Therefore, the objectives of this study were 1) to assess internal medicine residents' perceptions of the participation of clinical pharmacists during rounds, 2) to evaluate which types of recommendations internal medicine residents prefer receiving from a clinical pharmacist on rounds, and 3 ) to examine internal medicine residents past experiences with clinical pharmacists on rounds.

\section{Methods}

Prior to the initiation, the project received exempt status from both the hospital institution and the medical school Institutional Review Boards. All internal medicine residents and neurology interns (as these interns spend their first year working as internal medicine residents) at one medical residency program in Dayton, Ohio were invited to participate in the study. Participation was voluntary.

Internal medicine residents rotate through three hospitals in the Dayton, Ohio region during their residency. During the study period, residents had interactions with only two rounding clinical pharmacists, one critical care pharmacist at a community level one trauma center and one acute care/internal medicine pharmacist provider with a scope of practice at a Veterans Affairs hospital. The 4-week internal medicine rotation at the Veterans Affairs hospital consisted of four resident teams. There were 2 attendings who each oversaw 2 teams. Each team had a senior resident, an intern, and medical students.

The Interactions with Pharmacists on Rounds survey was developed using peer-reviewed literature. The survey was created to assess the individual's perceptions of and experiences with clinical pharmacists in the rounding setting. The survey contains 7 items about the individual's past experiences with pharmacists on rounds (5-point Likert-type scale; $1=$ Strongly Disagree, $5=$ Strongly Agree), 2 items about preferred recommendations from pharmacists on rounds (ranking questions), and 6 items about the individual's perceptions of pharmacy practice (5-point Likert-type scale; 1=Strongly Disagree, 5=Strongly Agree). Additional demographic questions were included to assess the respondent's year of residency and gender.

The Interactions with Pharmacists on Rounds survey was entered into Qualtrics and emailed to the internal medicine residents and neurology interns by their residency program coordinator. No identifiable information was obtained during this process. A reminder email to encourage survey completion was sent out at 2 and 4 weeks, as well.

Data were collected online via Qualtrics. After being exported from Qualtrics into Microsoft Excel, frequencies were performed on all items.

\section{Results}

\section{Demographics}

The survey was emailed to 80 medical residents; 27 residents completed the study (33.75\% response rate). Participation was evenly split amongst the three years of residency classes, with each year of residency having 9 participants. Most participants were male $(\mathrm{N}=17,63.0 \%)$.

\section{Perceptions of Pharmacy Practice (Table 1)}

Internal medicine residents agreed that both pharmacists and physicians should contribute to decisions regarding the type and dosage of medications being ordered (Mean \pm SD $=4.36 \pm$ 0.88 ) and that clinical pharmacists should clarify a physician's order when they believe it could have detrimental effects to the patient (Mean $\pm S D=4.96 \pm 0.20$ ). They felt neutral about whether or not clinical pharmacists have the skill level necessary to be licensed as a mid-level practitioner with independent prescribing rights (Mean $\pm S D=3.00 \pm 1.30$ ) and also felt neutral regarding their level of understanding of the education and training process of pharmacists (Mean $\pm \mathrm{SD}=$ $3.77 \pm 1.05)$.

\section{Past Experiences with Rounding Pharmacists (Table 1)}

The internal medicine residents responded favorably regarding their past experiences with rounding clinical pharmacists. Most residents strongly agreed that clinical pharmacists are a reliable source of both general and clinical drug information on rounds (Mean \pm SD $=4.89 \pm 0.42,4.93 \pm 0.26$ respectively). The residents strongly agreed that they always want a pharmacist to be a part of their rounding team (Mean $\pm S D=4.93 \pm 0.26$ ) and that interacting with rounding clinical pharmacists has improved their drug knowledge (Mean $\pm S D=4.74 \pm 0.44$ ).

\section{Preferred Recommendations}

Overall, the internal medicine residents who participated in the study appreciate recommendations from rounding clinical pharmacists on topics such as antibiotic stewardship, anticoagulants, and renal dose adjustments (Figure 1 ) in person before, during, or after rounds (Figure 2).

\section{Discussion}

In general, internal medicine residents who participated in this survey had positive past experiences with rounding clinical pharmacists, which matches the results of current literature on the experiences of physicians with clinical pharmacists..$^{5-7}$ The residents have witnessed clinical pharmacists on rounds working to resolve drug-related issues, being a reliable source of general and clinical drug information, and informing them about clinical issues with medication orders. These residents 
felt strongly that they want a clinical pharmacist to always be a member of their rounding team. Likely, the residents understand the value of a rounding clinical pharmacist being involved in the care of their patients, which ultimately leads to an improvement in patient outcomes. ${ }^{10}$

Internal medicine residents who responded to the survey most appreciate receiving recommendations in person, as well as before, after, or during rounds. One study in Australia found that, as a large component of a clinical pharmacist's work includes pointing out others' errors and requesting physicians to do corrective work, it is important for rounding pharmacists to build and maintain relationships with these providers. ${ }^{11}$ Strong relationships are likely better developed face-to-face, which may be the reason why the internal medicine residents preferred to receive recommendations from rounding pharmacists in an in-person manner. The internal medicine residents most appreciate receiving recommendations on topics such as anticoagulants and antimicrobial stewardship. It is significant that these residents value pharmacists' input on these topics, as evidence has shown that pharmacist-managed inpatient warfarin reduces excessive anticoagulation, major drug interactions, and time spent at supratherapeutic international normalized ratios and that pharmacist recommendations on antibiotic choice, dosing, or administration route leads to a decreased length of stay and increased cost savings for medical centers. ${ }^{12}$

In regard to their perceptions of pharmacy practice, the internal medicine residents strongly agreed that clinical pharmacists should clarify orders with physicians and that pharmacists and physicians should be viewed as collaborators and colleagues. These results mirror what the literature shows regarding physicians' perceptions of rounding pharmacists. ${ }^{5-7}$ The positive perceptions are promising for the future of clinical pharmacy, as upon completion of their residency, these physicians may be more likely to positively view clinical pharmacists and ask for their involvement in the rounding process. The internal medicine residents felt neutral about whether or not clinical pharmacists have the skill level necessary to be licensed as midlevel practitioners with independent prescribing rights. There are likely a multitude of reasons for this belief that were not examined in this study, but it could be due to the fact that the residents do not have a strong understanding of the education and training process of pharmacists. Continuing to inform internal medicine residents about the education and training that pharmacists receive could open the door for physicians to advocate for additional clinical pharmacy services in the near future.

Limitations of this study include the generalizability, because the survey was sent out to a small sample size of residents in one internal medicine residency program. The results could be expanded upon by conducting this study through multiple residency programs. This was a non-validated, survey-based study that relied on voluntary participation, and a low response rate may have influenced the results. The term clinical pharmacist was used throughout the survey but not defined for the survey participants; therefore, participants may have interpreted this terminology differently. The survey may not have included a complete list of topics that the resident preferred to receive recommendations on and therefore may be missing topics that the resident would prefer more. There was the potential for bias, as residents who have positive perceptions of pharmacists on rounds may have been more likely to complete the survey. Additionally, there may have been social desirability bias with residents attempting to minimize undesirable traits and maximize desirable ones for the research team. Future research can aim at developing and implementing methods to improve medical residents' knowledge of pharmacist training and education, as well as finding ways to encourage and strengthen the relationships between rounding pharmacists and resident teams.

\section{Conclusion}

Internal medicine residents have had positive past experiences with rounding acute care clinical pharmacists and strongly agreed that they always want a clinical pharmacist on their rounding team. They most prefer receiving recommendations from clinical pharmacists in person before, during, or after rounds and most appreciate recommendations on the topics of anticoagulation, antimicrobial stewardship, and renal dose adjustments. The residents did not express a strong knowledge of pharmacists' education and training process, which may have led to their lack of agreement that pharmacists are equipped to be mid-level practitioners.

\section{Conflicts of Interest: None}

\section{Funding: None}

Acknowledgements: This material is the result of work supported with resources and the use of facilities at the Dayton Veterans Affairs Medical Center. The contents do not represent the views of the U.S. Department of Veterans Affairs or the United States Government. 


\section{References}

1. American College of Clinical Pharmacy. About Clinical Pharmacists. Accessed March 16, 2021.

https://www.accp.com/about/clinicalpharmacists.aspx.

2. Leape LL, Cullen DJ, Clapp MD, et.al. Pharmacist participation on physician rounds and adverse drug events in the intensive care unit. JAMA. 2000;282(3):267-270

3. Bjornson DC, Hiner WO, Potyk RP, et.al. Effect of pharmacists on health care outcomes in hospitalized patients. Am J Hosp Pharm. 1993;50(9):1875-1884

4. Patel NP, Brandt CP, Yowler CJ. A prospective study of the impact of a critical care pharmacist assigned as a member of the multidisciplinary burn care team. J Burn Care Res. 2006;27(3):31-313

5. Abdel-Latif MMM. Hospital doctors' views of, collaborations with and expectations of clinical pharmacists. Eur J Hosp Pharm Sci Pract. 2017;24(6)

6. Makowsky MJ, Schindel TJ, Rosenthal M, et.al. Collaboration between pharmacists, physicians, and nurse practitioners: A qualitative investigation of working relationships in the inpatient medical setting. $J$ Interprof Care. 2009;23(2):169-184

7. Khdour MR, Alayasa KW, AlShahed QN, Hawwa AF. Physicians' perceptions, attitudes and expectations regarding the role of hospital-based pharmacist in West Bank, Palestine. Int J Pharm Pract. 2013;21(3):178-84

8. Wolfe ME, Moeller KE, Woods B. Medical students' perceptions on the role of pharmacists. J Pharm Pract. 2018;31(6):623-628

9. Smith WE, Ray MD, Shannon DM. Physicians' expectations of pharmacists. Am J Health-Syst Pharm. 2002;59:50-57

10. Viktil KK, Blix HS. The impact of clinical pharmacists on drug-related problems and clinical outcomes. Basic Clin Pharmacol Toxicol. 2008;102(3):275-280

11. Rixon S, Braaf S, Williams A, Liew D, Manias E. Pharmacists' interprofessional communication about medications in specialty hospital settings. Health Communication. 2015;30:1065-1075

12. Kaboli PJ, Hoth AB, McClimon BJ, et.al. Clinical pharmacists and inpatient medical care: A systematic review. Arch Intern Med. 2006;166(9):955-964 
Table 1: Interactions with Pharmacists on Rounds

\begin{tabular}{|c|c|}
\hline Item & Mean \pm SD \\
\hline \multicolumn{2}{|l|}{ Perceptions of Pharmacy Practice } \\
\hline $\begin{array}{l}\text { 1. Pharmacists should clarify a physician's order when they feel that it might have } \\
\text { detrimental effects on the patient. }\end{array}$ & $4.96 \pm 0.20$ \\
\hline $\begin{array}{l}\text { 2. Both pharmacists and physicians should contribute to decisions regarding the type and } \\
\text { dosage of medicine given to a patient. }\end{array}$ & $4.36 \pm 0.88$ \\
\hline 3. A physician should be viewed as a collaborator and colleague with a pharmacist. & $4.77 \pm 0.42$ \\
\hline 4. I have a good understanding of the education and training process of pharmacists. & $3.77 \pm 1.05$ \\
\hline $\begin{array}{l}\text { 5. Clinical pharmacists have the skill level necessary to be licensed as a mid-level practitioner } \\
\text { (i.e. nurse practitioner, physician assistant) with independent prescribing rights. }\end{array}$ & $3.00 \pm 1.30$ \\
\hline $\begin{array}{l}\text { 6. If clinical pharmacists are to be mid-level practitioners, they should complete additional } \\
\text { training (i.e. residency) in order to do so. }\end{array}$ & $4.38 \pm 0.79$ \\
\hline \multicolumn{2}{|l|}{ Past Experiences } \\
\hline $\begin{array}{l}\text { 1. In my experience on rounds, pharmacists are a reliable source of general drug information } \\
\text { (e.g., specific facts about drugs which can be found in standard references). }\end{array}$ & $4.89 \pm 0.42$ \\
\hline $\begin{array}{l}\text { 2. In my experience on rounds, pharmacists are a reliable source of clinical drug information } \\
\text { (e.g., information regarding the clinical use of drugs in specific situations). }\end{array}$ & $4.93 \pm 0.26$ \\
\hline $\begin{array}{l}\text { 3. Pharmacists routinely inform me on rounds if they discover clinical problems with my } \\
\text { orders. }\end{array}$ & $4.63 \pm 0.95$ \\
\hline $\begin{array}{l}\text { 4. I was fully informed as to the role of the pharmacist on my rounding team at the beginning } \\
\text { of my residency. }\end{array}$ & $4.11 \pm 1.13$ \\
\hline 5. I want a pharmacist to always be a member of my rounding teams. & $4.93 \pm 0.26$ \\
\hline $\begin{array}{l}\text { 6. When a pharmacist makes recommendations to me on rounds, I feel as though my } \\
\text { knowledge is being threatened. }\end{array}$ & $1.23 \pm 0.44$ \\
\hline 7. Through my encounters with pharmacists on rounds, my drug knowledge has improved. & $4.74 \pm 0.44$ \\
\hline
\end{tabular}

5-point Likert-type scale; 1=Strongly Disagree, 5=Strongly Agree 
Figure 1: Ranking of Recommendation Topic Preferences

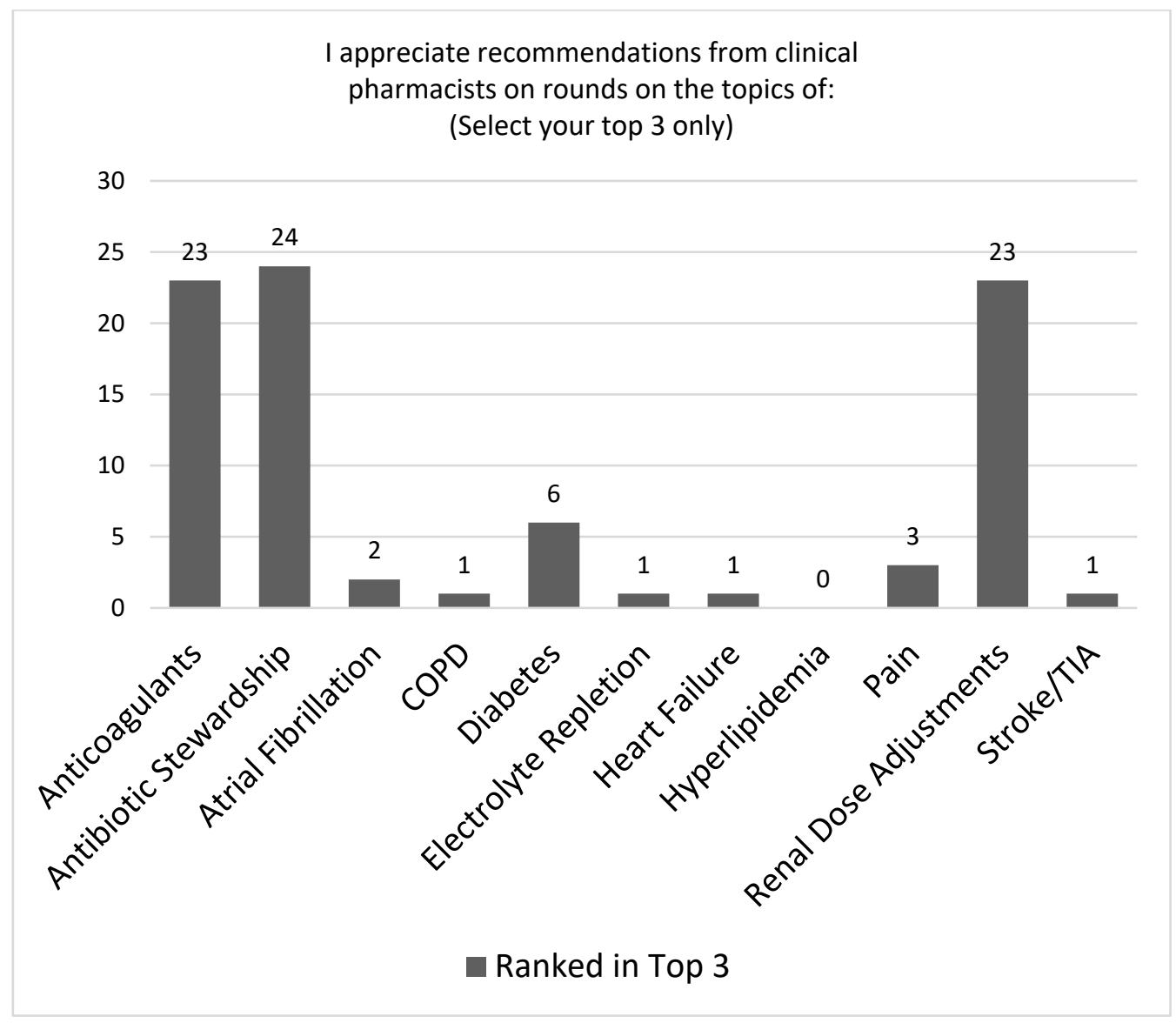


Figure 2: Contact Preferences

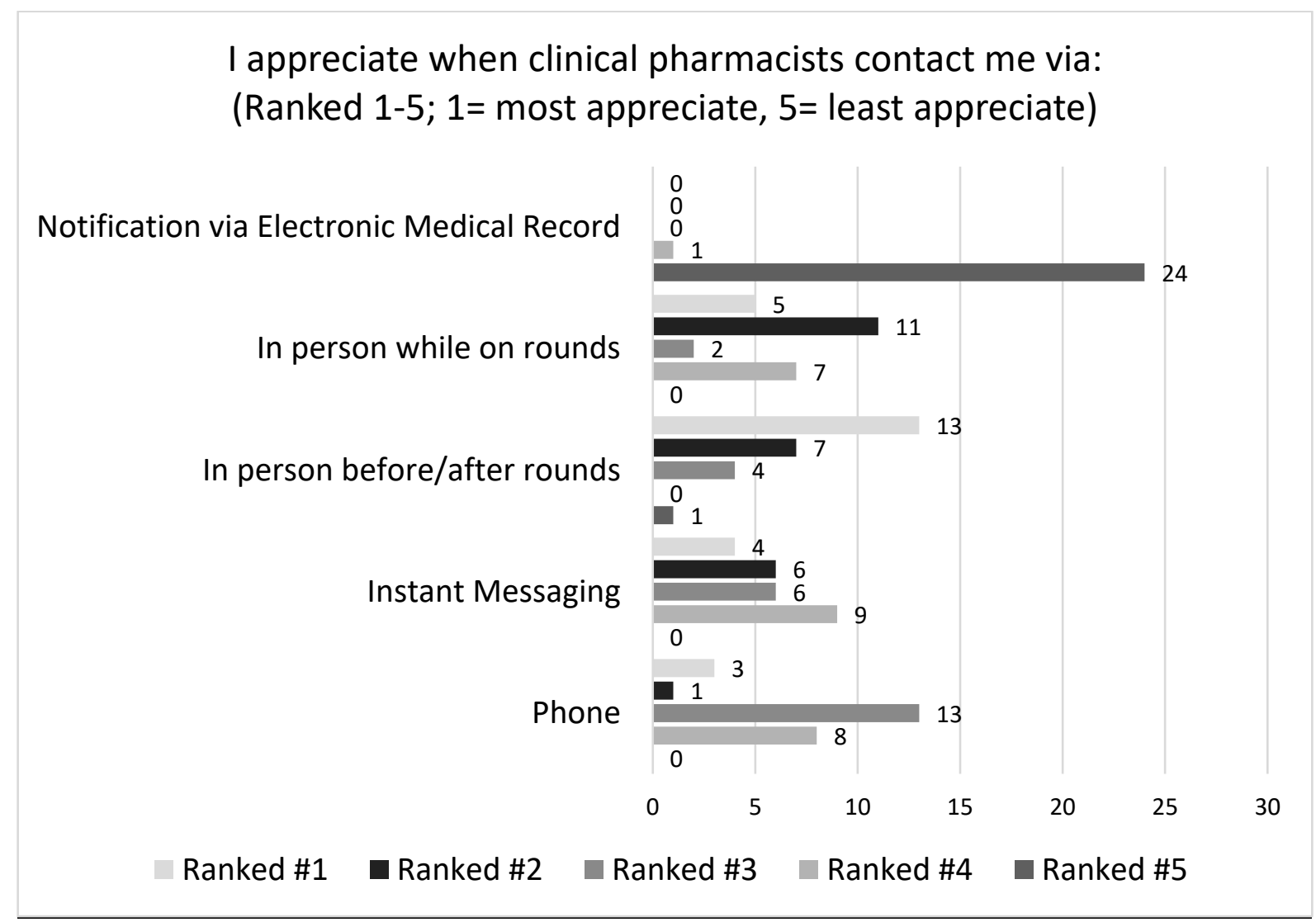

